\title{
Doping-Induced Layered Structure in $N$-Alkylated Polyanilines
}

\author{
Wen-Yue Zheng, ${ }^{*}$ Kalle Levon, ${ }^{* \dagger} \dagger$ Tapani TAKa, ${ }^{* *}$ \\ Jukka LAAKSO, ${ }^{* *}$ and Jan-Eric ÖSTERHOLM** \\ * Department of Chemistry, Chemical Engineering and Materials Science, Polytechnic University, \\ Six MetroTech Center, Brooklyn, New York, N.Y. 11201, U.S.A. \\ ** Neste Oy Corporate $R \& D, S F-06150$, Kulloo, Finland
}

(Received September 22, 1995)

\begin{abstract}
The influence of various organic dopants on the structure of $N$-alkylated polyanilines substituted with alkyl side chains of different lengths and at different degrees of alkylation has been studied by FT-IR, UV-Visible spectroscopies, and X-ray diffraction. The results show that the protonation as seen for polyaniline emeraldine base can be achieved successfully through the doping method in our experiments. The conductivities of the doped polymers decrease with increasing the length of side chains and the degree of alkylation. The polyanilines doped with methanesulfonic acid (MSA), toluenesulfonic acid (TSA), and dodecylbenzenesulfonic acid (DBSA) induce a co-operative effect with the alkyl side chains on the formation of layer order. Small dopants distort the layered structure and prevent the crystallization of the side chains. The interaction of the alkyl side chains and alkyl group in DBSA assist in the development of the layered structure.

KEY WORDS

Polyaniline / Doping / Layer Formation /
\end{abstract}

Polyaniline has been recognized as an interesting conducting polymer though its intractability has limited the comprehensive understanding of its structure as well as the potential applications. Recently, it has been found that this barrier can be overcome by the incorporation of flexible side chains to the stiff backbones leading to highly soluble polyanilines. The resulting polyanilines are fusible or soft below the temperatures where crosslinking or thermal decomposition prevails. ${ }^{1,2}$ Furthermore, these alkyl substituents induce the formation of a layered structure, side chain crystallinity, mesophase, and thermochromic behavior. ${ }^{3}$ As the side chains in the layered structure "melt" or disorder, the sheets consisting of a parallel array of main chains can quickly respond to external shear, which renders this layered structure significant for melt or solution processing. Although a relatively large volume of alkyl side chain segments has been added to polyaniline, the substituted main chains still retain the basic conjugated structure and basic optical properties.

The control of the properties of the conducting polymers by the process of doping is currently under intense scrutiny. ${ }^{4,5}$ Doping not only initiates an increase in conductivity of the initially neutral conjugated polymer, but also leads to remarkable changes in proccessability, morphology, and mechanical properties. ${ }^{6-8}$ Recently, an important innovation by the use of surfactants with polyaniline has attracted great attention in the academic and industrial research community. ${ }^{9,10}$ Since these dopants, such as dodecylbenzene sulfonic acid (DBSA) and camphorsulfonic acid (CSA), can perform as plasticizers, the doping of intractable polyaniline creates soluble and processable forms of the parent polymers. The complex of polyaniline and DBSA has been found to form a well-defined layered, smectic-like structure in which the alkyl chains function as spacers between parallel stacks of the polymer backbones. ${ }^{11}$ From the experimental and theoretical analyses, the individual polyaniline chains doped with these dopant-ions showed enhanced stiffness due to electronic repulsion and steric crowding of counterions as proposed by Fredrickson. ${ }^{12}$ The enhanced chain rigidity and persistence length can be responsible for lyotropic liquid crystallinity seen for PANi/CSA in $m$-cresol solution by Cao and Smith. ${ }^{13}$

The importance of main chain alignment, especially in strech-oriented materials, for enhancement of specific electronic structural properties has been widely recognized. ${ }^{14}$ In a single isolated polymer chain, conduction only occurs along the polymer chain axis. For the threedimensional structure of the polymer, the transport of charge carriers occurs not only along the alignment direction but also between the neighbor chains by the hopping mechanism. ${ }^{15}$ The structural order in an intrinsically conducting polymer can have a pronounced influence on its charge-transport characteristics. ${ }^{16}$ High anisotropy has been usually achieved through stretch orientation, appropriate solvent casting, thermal treatment or deposition of Langmuir-Blodgett multilayers. ${ }^{8,17}$ It has been reported that the doped polyanilines with surfactant (CSA and DBSA) exhibit an increase in conductivity of $10^{3}$ in contrast to $\mathrm{HCl}$-doped PANi due to an enhanced ordering as well as possible conformational changes. ${ }^{18}$

Evidently, conducting polymers with ordered structure have the advantage of a combination of high conductivity with good solubility properties. Previously, we have used alkylation to induce layered structures in PANi with the control of the side chain length and degree of alkylation. In this paper, we will apply three organic acids of different size of substituents to dope alkylated PANis. Our goals are to discuss how structure perfection of the layers of alkylated PANi backbones can be enhanced by dopants, in particular by dopants with long alkyl tails; how side chain ordering can be disturbed by doping; and how perfection of the layer ordering and the extent of doping should depend upon the interaction between the side chain and the dopants. ${ }^{19}$

\footnotetext{
† To whom correspondence should be addressed.
} 


\section{EXPERIMENTAL}

The neutral $N$-alkylated polyaniline used in this work was prepared via deprotonation of the amine sites in polyaniline leucoemeraldine followed by alkylation with different alkyl substituents. ${ }^{3} 150 \mathrm{ml}$ DMSO and 0.0269 mol of $\mathrm{NaH}$ were added into a $250 \mathrm{ml}$ nitrogen-flushed reaction flask equipped with magnetic stirring. The mixture was heated up to $50-60^{\circ} \mathrm{C}$ and stirred until $\mathrm{NaH}$ was dissolved. $0.0215 \mathrm{~mol}$ of polyaniline leucoemeraldine (PANi-LB) was added to NaH/DMSO solution at $60^{\circ} \mathrm{C}$ and the metalation reaction was continued for $4 \mathrm{~h}$. The resulting viscous solution was cooled to $40^{\circ} \mathrm{C}$ and $0.0215 \mathrm{~mol}$ of $n$-alkyl bromide was dropped in. The reaction was continued for $16 \mathrm{~h}$ and the blue sticky precipitate developed. The precipitate was extensively washed with methanol, water and acetone alternately to remove all unreacted species. The green fiber-like materials was obtained by pouring the solution of the precipitate in tetrahydrofuran (THF) to $0.5 \mathrm{M}$ aqueous $\mathrm{HCl}$, washed by water and acetone and was then neutralized in $\mathrm{NH}_{3} \cdot \mathrm{H}_{2} \mathrm{O}$.

The products were purified before characterization or futher usage. The degrees of substitution in these samples have defined previously. The homogeneous doping of the polymers were accomplished by slowly precipitating the polymer THF solutions into $0.5 \mathrm{M}$ methanesulfonic acid (MSA) (Tokyo Kasei), toluenesulfonic acid (TSA) (Aldrich), and dodecylbenzenesulfonic acid (DBSA) (Tokyo Kasei) aqueous solutions. The green precipitates developed in the process were washed several times by deionized water to remove the extra dopants free out of the polymer chains and finally dried by high vacuum at room temperature. The degrees of doping were based on the weight changes before and after doping. Reproducibility of doping and error range in the doping process was estimated to be below $10 \%$.

Infrared spectra were recorded with a Perkin Elmer 1600 FT-IR spectrometer connected with a PC computer with Spectrum Calculation program (SC). Spectra were taken of neat films on $\mathrm{KBr}$ plates under a purge of nitrogen gas. UV spectra were obtained on a Varian $100 \mathrm{UV}$-Visible spectrometer. Thin films of polymers were prepared on a flat quartz surface from dilute THF solution for the solid state measurements.

A Philips APD 3720 X-ray generator controlled by a computer was used for the WAXD experiments. The point focus beam was monochromatized to $\mathrm{Cu}-K$ with a graphite crystal. X-Ray diffraction data were recorded by a diffractometer. The X-ray source, a XRG 3100 generator utilized $\mathrm{Cu}-K$ target $\left(l_{K}=1.5406 \AA\right)$ with generator settings $40 \mathrm{kV}$ and $20 \mathrm{~mA}$. The 2 step size for each individual data collection point was set into $0.04^{\circ}$ and the time of remaining on every point was limited to $50 \mathrm{~s}$. The measurements were performed by utilizing the grinded samples as doping-precipitation or thick films cast from doped polymer THF solution.

The electrical conductivities of the samples in the form of thick films or pellets made by compressing of precipitate were measured using both the standard fourprobe and two-probe techniques.

\section{RESULTS AND DISCUSSION}

\section{Emeraldine Salts with Various Dopants}

The structural change of polyaniline during doping corresponding to the change of $\mathrm{pH}$ and redox potential has been illustrated in the literature before. ${ }^{10,15}$ The undoped emeraldine base has an amorphous structure. On the other hand, its salt after protonation with acids has been previously reported to show a semicrystalline texture with the crystal phase exhibiting a pseudoorthorhombic unit cell. ${ }^{20}$ The two distinctive diffraction peaks have been defined as to (100) and (110) respectively by Pouget $e t$ al. ${ }^{20}$ In this work, the PANi-EB becomes partially crystalline as shown in Figure 1 when doped with MSA and TSA. The scattering patterns consist of one broad reflection centered around $20-21$ corresponding to amorphous region, a strong reflection at $20-27(d \sim 3.46 \AA)$ and some weak features.

In the X-ray patterns of PANiEB-DBSA, an additional pronounced feature appears at small angle between $20-25 \quad(d=27.7 \AA)$. This is a characteristic feature of an interlayer repeat. The polymer forms a layered structure in which the alkyl tails of counterions function as spacers between parallel planes or sheets stacked by polymer backbones. The PANi/DBSA complex has also been shown to be birefringent under cross polarized light.

\section{$N$-Alkylated PANi Salts with Various Dopants}

Changes of Chemical Structure upon Doping. The slow precipitation of the THF solutions into stirred aqueous acid media was carried out in effort to achieve optimal protonation of the aniline units. PANi/MSA and PANi/ TSA complexes have limited solubilities but PANi/DBSA complex salts are soluble in the weakly polar solvents, such as THF, dioxane, chloroform, and toluene. The resulting solutions appear with clear green color and the polymers reverse to the neutral state with blue color when organic bases, like pyridine or triethylene amine are added. The fact that these polymers in the doped state have good solubility and are stable in the solution is impressive because most of the conducting polymers precipitate upon doping their solutions. ${ }^{21}$

\section{UV-Visible Absorption}

The characteristic features of UV-Visible absorptions for neutral PANiEB-EB (emeraldine base) in an appropriate solvent, NMP or DMSO or in solid state as a thin

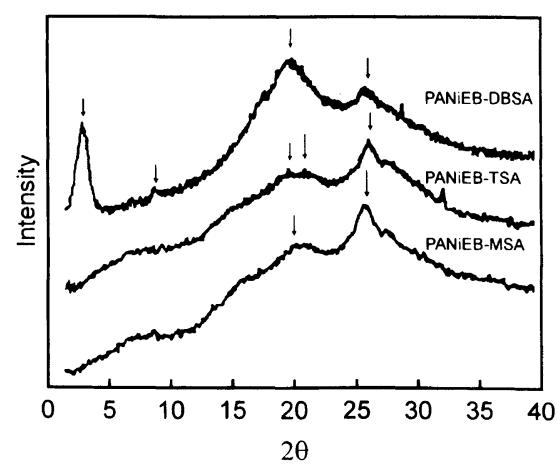

Figure 1. X-Ray diffraction patterns of polyaniline emeraldine (PANiEB) doped by MSA, TSA, and DBSA. 

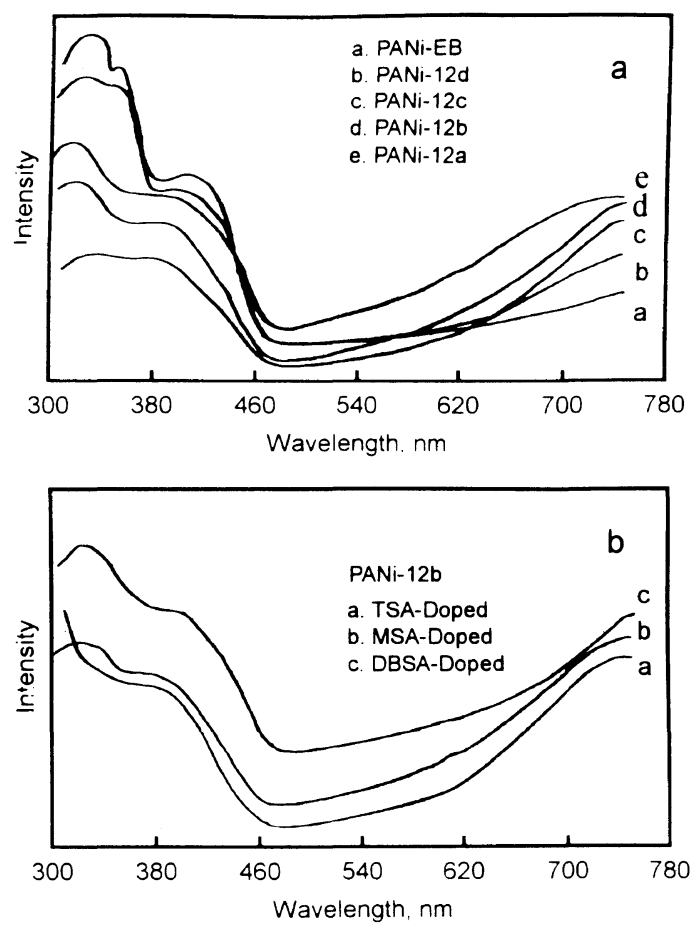

Figure 2. UV-visible spectra of (a) solid thin film polyanilines with various degrees of $N$-dodecylation doped by methanesulfonic acid (MSA); (b) PANi-12b doped by different dopants.

film are the two absorptions at $600 \mathrm{~nm}$ and $320 \mathrm{~nm}$. As generally suggested, the former absorption is due to an electron transition on quinoid rings and the latter absorption is due to $\pi-\pi^{*}$ transition related to the benzenoid form. ${ }^{21,22}$ The spectra in our previous study have shown that the partial $\mathrm{N}$-alkylation of the polyaniline allow the retention of the coexistence of these two basic forms. Dodecylated polyanilines with a different degree of alkylation (sample $\mathrm{c}$ is with highest extent of alkylation) are used in the first set of these doping experiments. ${ }^{3}$ As depicted in Figure 2a, thin films of alkylated polyanilines doped by methanesulfonic acid (MSA) display an absorption identical to the emeraldine base doped by $\mathrm{HCl}$. The absorption at $440 \mathrm{~nm}$ and the low energy transition located at wavelength longer than $780 \mathrm{~nm}$ have been related polaron formation. The UV absorption for MSA-doped polyanilines with different degrees of substitution are given in Figure 2a. With increase of $N$-alkylation, the transition at $440 \mathrm{~nm}$ decreases gradually in intensity, and the one at $600 \mathrm{~nm}$ shifts to low wavelengths above $800 \mathrm{~nm}$. The spectra for PANi12b dopted with three different dopants show the same absorptions in the $300-780 \mathrm{~nm}$ range in Figure $2 \mathrm{~b}$. The protonation with the other dopants induces similar changes in the absorption spectrum.

\section{FT-IR Spectroscopy}

The variation of molecular structure upon doping was also defined by FT-IR measurements for the doped samples. A preliminary assignment has been given to the IR spectra of polyaniline leucoemeraldine and neutral substituted derivatives. ${ }^{3}$ Figure 3 shows the FT-IR spectra for the $N$-alkylated polyanilines with the different side chains upon doping by MSA. The new broad peak appearing centered at $3200 \mathrm{~cm}^{-1}$ represents the formation of $\mathrm{NH}$ bonds due to the protonation of nitrogen.

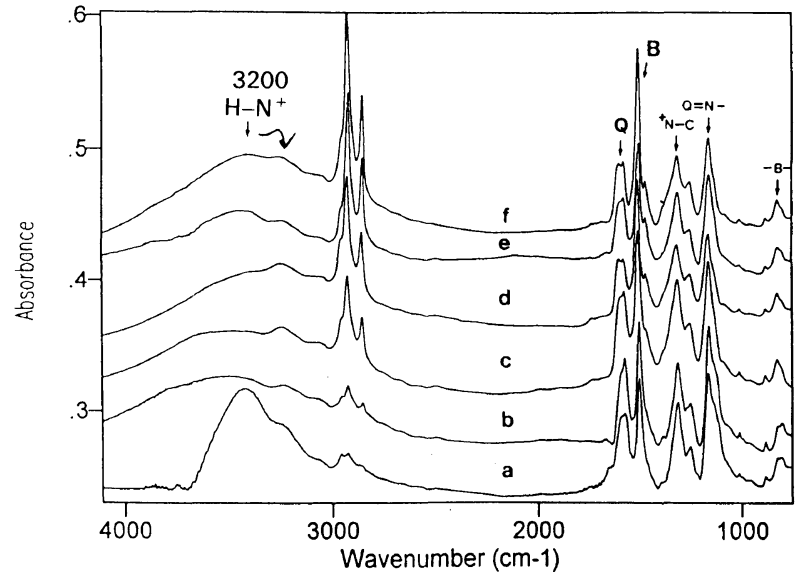

Figure 3. FT-IR spectra of $N$-alkylated polyaniline with different lengths of side chain after MSA-doping: A, PANi-4; b, PANi-8; c, PANi-12a; d, PANi-14; e, PANi-16; f, PANi-18.

Table I. Doping level of $N$-alkylated polyanilines upon organic acids

\begin{tabular}{|c|c|c|c|c|}
\hline \multirow{2}{*}{ Polymers } & \multirow{2}{*}{$\begin{array}{l}\text { Degree of } \\
\text { alkylation }^{\text {a }}\end{array}$} & \multicolumn{3}{|c|}{ Percentage of doped aniline units } \\
\hline & & MSA & TSA & DBSA \\
\hline PANi-EB & 0 & 39.8 & 23.2 & 23.0 \\
\hline PANi-4 & 83.3 & 30.0 & 24.2 & 18.2 \\
\hline PANi-8 & 74.5 & 27.6 & 16.1 & 23.4 \\
\hline PANi-12d & 21.6 & 28.2 & 23.4 & 26.2 \\
\hline PANi-12c & 37.9 & 23.7 & 19.3 & 24.5 \\
\hline PANi-12b & 59.0 & 20.9 & 20.9 & 23.1 \\
\hline PANi-12a & 76.8 & 23.4 & 16.7 & 25.9 \\
\hline PDAN & 100 & 10.2 & 8.7 & 14.5 \\
\hline PANi-14 & 76.3 & 18.5 & 14.8 & 23.2 \\
\hline PANi-16 & 72.5 & 15.3 & 16.4 & 26.3 \\
\hline PANi-18 & 67.5 & 22.8 & 18.7 & 27.8 \\
\hline
\end{tabular}

${ }^{a}$ Defined by elemental analysis. ${ }^{3}$ b Calculated from the mass changes of the samples after doping.

The band at $1600 \mathrm{~cm}^{-1}$ related to stretching vibration in quinoid form becomes broad and splits to two peaks at low frequency 1585 and $1565 \mathrm{~cm}^{-1}$. The band at $1496 \mathrm{~cm}^{-1}$ related to the stretching vibration in benzenoid form spontaneously shifts to low frequency by $10 \mathrm{~cm}^{-1}$. The intensity ratio of $1600 / 1490 \mathrm{~cm}^{-1}$ absorbencies was found dependent on the size of side chains substituted on aniline backbone. The bands at 1318 and $1140 \mathrm{~cm}^{-1}$ grow and become strong compared to the spectra of neutral samples. ${ }^{23}$ Because the protonation of nitrogen sites causes the redistribution of the electrons between phenyl ring and nitrogen, these modes are derived from the vibration of charged $\mathrm{CN}$, NC, or adjacent $\mathrm{CH}$ bonds. The basic features of the spectra evidence that protonation occurs in a similar fashion with all the dopants but is independent on the type of the dopants.

Extent of Doping. Doping levels (percentage of aniline molar units protonated by acid molecules) calculated from the mass changes upon doping are summarized in Table I. The smallest degree of doping $(10 \%)$ was found for PDAN, the fully substituted polyaniline (polymerized from the alkylated monomer ${ }^{3}$ ) and the highest degree of doping with the EB, the unsubstituted polymer $(35 \%)$. The degree of doping with MSA decreases with the 


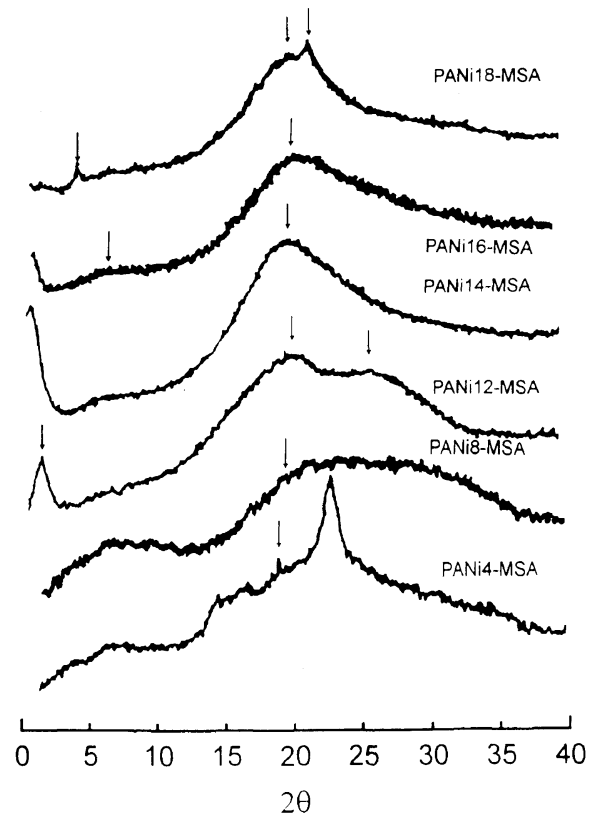

Figure 4. X-Ray diffraction patterns of $N$-alkylated polyanilines doped by MSA.

increasing length of the side chains from $30 \%$ for butyl susbstituted to $15 \%$ for hexadecyl substituted ones. With the use of TSA, the degree of doping lies between $15-18 \%$ independent on the size of the substituents. DBSA causes an initial drop to $18 \%$ with butyl substituents and then increases to $27 \%$ with the longest side chains. This may be due to geometrical stabilization resulted from hydrophobic interaction between alkyl side chains and the dodecyl substituents of the dopant. ${ }^{24}$ One has to be careful with a deeper analysis of the degree of doping although these trends would indicate above discussion because the error range in the experiments may be large.

Ordering of N-Alkylated PANi Salts with Various Dopants

$X$-Ray Diffractions of Doped N-Alkylated PANis. With the covalent incorporation of short butyl side chains, the $\mathrm{N}$-butylated polyaniline-methanesulfonic salt (PANi4MSA) remains in a semicrystalline form with the diffractions (100) and (110) of enlarged $d$ spacings $4.70 \AA$ and $3.94 \AA$ as seen in Figure 4. The expansion of the cell can be explained in term of the increase of the interchain separation due to butyl substituents. The pattern for $\mathrm{N}$ octylated PANi8-MSA salts shows an only broad amorphous feature, and octylation has prevented the formation of the pseudo-orthorhombic crystal. PANi12 and PANil4 in the MSA doped salt exhibit intense reflections at small angles accompanied with broad scattering peaks at the wide 2 angle $\sim 19-22^{\circ}$. The wide angle peak may be the superposition of the amorphous scattering and disordering of the alkyl side chains. The peaks located at small angle are indicative of a layered structure. The small angle diffraction originating from the separation between the main chains seems shift to lower angels with the increase of the side chain length. For PANi16-MSA complex, the small angle diffraction is hardly observed anymore. Similar case is observed with PANi18-MSA salt from, but in this case the weak diffractions at low angle $\left(5-8^{\circ}\right)$ region indicative of higher order reflections.

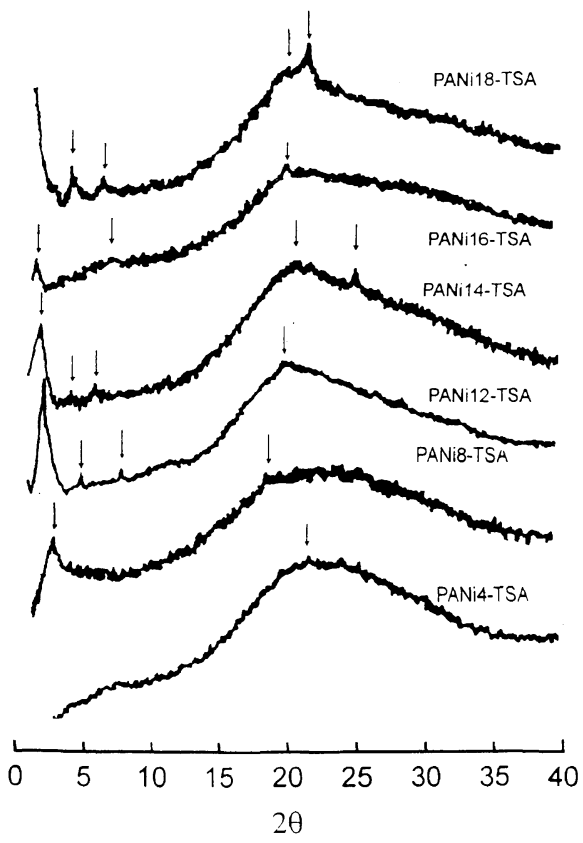

Figure 5. X-Ray diffraction patterns of $N$-alkylated polyanilines doped by TSA.

Thus it is assumable that the main diffraction is already out of the range in the experiments.

Interestingly, a strong diffraction at $22^{\circ}$ is seen for this complex. It is typical for the samples with long alkyl side chains that the interdigitation of these alkyl chains results in the formation of hexagonal paraffinic crystal, which now has not been disturbed by the presence of the dopant. In general, doping of the MSA results in the formation of pseudo-orthorhombic crystalline form. Butylation causes expansion of this cell structure, octylation prevents the formation of this type of crystallinity resulting in only amorphous material, and for alkylation with longer side chains, the alkyl side chains organize the complex in a layered form. The crystalline packing of the side chain is possible with side chain of 18 carbons.

Toluene sulfuric acid, TSA as the dopant with EB resulted also in the formation of pseudo-orthorhombic crystals. With butylation in PANi4-TSA complex, no crystalline ordering is achieved as the X-ray pattern and exhibits only a broad typical halo for amorphous polymers as seen in Figure 5 without any indication of ordering. The TSA doped PANi8 salt forms the layered structure indicated by the reflection at the small angle region in the X-ray pattern. The relatively weak and broad peak $(d=25.5 \AA)$ of this salt can be related to imperfection of the layered structure. PANi 12 and PANi14 in TSA doped forms show the intense diffractions of small angels including weak peaks located at small angle range $\left(5-8^{\circ}\right)$. These combinations seem to be optimal conditions for the layering as the smallest angle peak is intensive and the weak peaks indicative of higher order (2nd and 3rd) reflections of layers. PANi16-TSA complex shows weak evidence for layering, but PANi18TSA again shows strong layering with also side chain crystallinity as observed by the wide angle diffraction. As was seen with MSA doping, TSA also prevents the formation of pseudo-orthorhombic crystals and with long alkyl side chains the layering becomes dominant. 


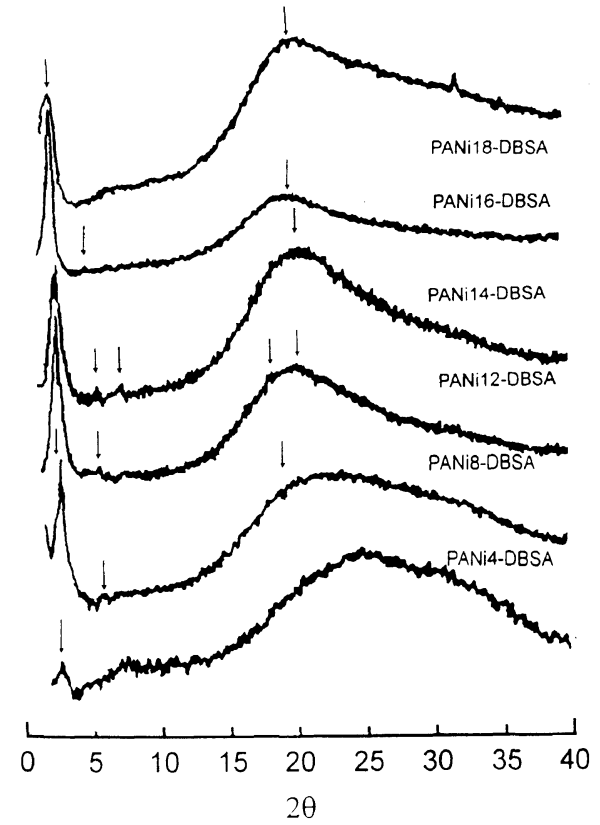

Figure 6. X-Ray diffraction patterns of $N$-alkylated polyanilines doped by DBSA.

This tendency with TSA was with shorter side chains than with MSA indicating that the size of the dopant has also an importance in this cooperative packing.

In all of the DBSA doped samples a diffraction at the small angle was observed as also in the non-alkylated case as shown in Figure 6. The reflection is weak for PANi4-DBSA salt showing that the layering is not as obvious as with all the other cases. The butylation may cause disordering probably due to the large difference in the sizes of the side chains and the substituents in the dopants. The most optimal combination for layering access to be in the case of PANil6-DBSA pair as qualitatively the ration of the small angle diffraction for ordering and the wide angle diffraction for amorphous material seems to be the highest. Side chain crystallinity is not observed in those PANi18 samples.

The following several trends can be observed from the above results.

(1) The alkylation prevents the formation of the orthorhombic crystal structure common to acid doped polyaniline. It is noticed only in the butylated sample with MSA, the smallest dopant in expanded dimensions.

(2) Amorphous material is obtained for PANi8MSA, PANi4-TSA, and the weakest order for PANi4 DBSA. This maybe related to the with finding with alkylated polythiophenes which showed for copolymers that disordering was the strongest as the difference in the lengths if the side chains in the two monomer units was the largest. This, the lack of ordering in these pairs maybe also be related to the size difference between the alkyl chain and the dopant.

(3) With long alkyl side chain, layering is observed with all the dopants. With the use of the alkylated dopant, DBSA, the distance between the chains is determined by the size of the dopant, when the number of the carbons is 12 or less as seen in Figure 7. As the number of carbons in the side chains above 12 , the parameters of the layer are determined by the covalently bound substituents for all the doped samples. The layer formation is due to the

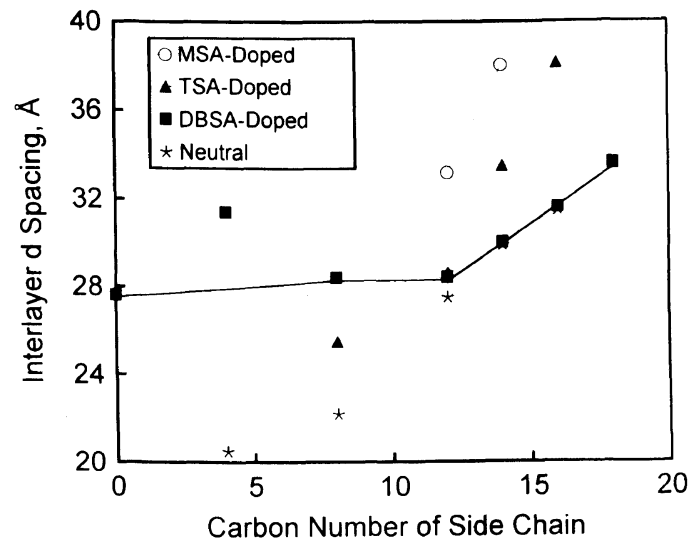

Figure 7. Variation of the $d$ spacing of the layer ordering reflections of neutral, MSA, TSA, and DBSA doped polyanilines as a function of the length of alkyl side chains.

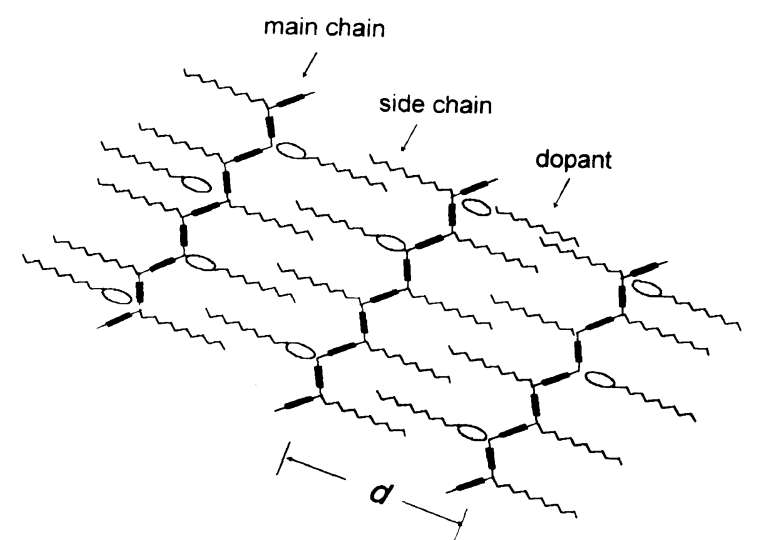

Figure 8. A model proposed for the layered structure built-up by polyaniline attached with flexible side chains and dopants.

repulsion between the non-polar chemical nature of side chains, and the polar chemical nature of charged aniline backbone. The insertion of dopant tailed with alkyl substituents in polyaniline chains makes the main chains more rigid and favors the layer orientation. The dopants enhance the formation of layers in polyaniline depending on the size of dopants counterion and by the insertion of dopant ions into the free space of the polymer chains with the covalently attached side chains. The number of associated dopants per monomer unit, geometric location of dopants and free volume provided by covalently bonded alkyl side chains should be taken into the cooperative stabilization of orientated structures.

The increment of the interlayered spacing per $\mathrm{CH}_{2}$ hydrocarbon units for MSA and TSA (Figure 7, 2.4 $\AA_{\text {) }}$ does not indicate an increase in the interdigitation with the increase of the length of the alkyl side chain, as the value for an ethylene unit in all-forms configuration is $2.52 \AA$. The same increment for DBSA doped samples is $1.5 \AA$ indicating an increase in the intercalation. The alkyl-substituents in the dopant seem to favor better intercalating structures as shows schematically in Figure 8. This optimal layering is probably due to the cooperative function including interactions between the alkyl side chains and the dopants. The scope of the interlayer $d$-spacing values in Figure 7 for the DBSA doped samples is analogous to that for the neutral alkylated polymers before doping. The fact that layer parameters are 
Table II. Average domain size of layered phase determined by using Debye-Scherrer equation

\begin{tabular}{lcccc}
\hline & \multicolumn{3}{c}{$L$ Domain size of layered order/A } \\
\cline { 2 - 5 } Polymers & Neutral & MSA-doped & TSA-doped & DBSA-doped \\
\hline PANi-EB & & & & 70 \\
PANi-4 & & & 52 & 28 \\
PANi-8 & & 56 & 82 & 88 \\
PANi-12a & 32 & 67 & 74 & 104 \\
PANi-14 & 42 & & 32 & 122 \\
PANi-16 & 14 & & & 92 \\
PANi-18 & & & & \\
\hline
\end{tabular}

identical before and after doping supports the fact that the dopant ions can be located in the vacant space formed by the adjacent alkyl chains and aniline backbone in the layered place without expanding the distance between the polymer chain. The hydrophobic interactions between the covalent chain and the alkyl chains in the dopant must be important for the formation of this intercalated layer as with the dopants without alkyl chain but which have a smaller overall size, the expansion in the layer was much larger.

Domain Size of the Ordered Structure. The domain size of the ordered phase, $L$ (coherence length), can be estimated from the width of the reflection peak at halfmaximum height using the following Debye-Scherrer formula. ${ }^{4,20}$

$$
L=\frac{0.9 \lambda}{\Delta(2 \theta) \cos \theta}
$$

where is the wavelength, is $1 / 2$ diffraction angle, and 2 means the width of the peak at half-maximum height. The resolution of the patterns in our X-ray experiments (= $=1.5406 \AA$ ) is set as $0.04^{\circ}$. The $L$ values deduced from the widths of the strong scatterings at small angle were utilized to evaluate the domain sizes of layered structure regions. The $L$ values for the polymers with the various lengths of side chains and size of dopants are summarized in Table II. Typically, $L$ ranges from 10 to $40 \AA$ for alkylated undoped polymers. The estimated values for doped samples scale from 30 to $130 \AA$, suggesting longer-range order induced by doping. The increase of $L$ value with the side chain length in DBSA-doped series indicates an increase of the domain size of layered phase. In particular, the sharpening of small-angle peak in DBSA-doped hexadecylated polyaniline and the largest $L$ value can be likely interpreted by the co-operation of alkyl side chain and alkyl tail of associated DBSA in length.

\section{Conductivity}

The initial goal in this study was to find the correlation between the aforementioned structural features and electrical properties. As listed in Table III, the resulting conductivities of the polymers doped with dopants of various sizes obtained from four-probe technique (measuring error within $10 \%$ ) are correlated with the length of the alkyl side chain and the extent of alkyl substitution. No matter what dopants used, the conductivities show a decreasing trend when the length of the side

Polym. J., Vol. 28, No. 5, 1996
Table III. Conductivity of the doped $N$-alkylated polyanilines with various organic acids

\begin{tabular}{lccc}
\hline & \multicolumn{3}{c}{ Conductivity $^{\mathrm{a}} / \mathrm{S} \mathrm{cm}^{-1}$} \\
\cline { 2 - 4 } Polymers & MSA & TSA & DBSA \\
\hline PANi-EB & 14.2 & 8.35 & 10.7 \\
PANi-4 & $1.19 \times 10^{-2}$ & $3.5 \times 10^{-3}$ & $5.54 \times 10^{-3}$ \\
PANi-8 & $6.18 \times 10^{-2}$ & $8.64 \times 10^{-4}$ & $3.12 \times 10^{-3}$ \\
PANi-12d & $6.13 \times 10^{-1}$ & $4.40 \times 10^{-3}$ & 2.06 \\
PANi-12c & $8.27 \times 10^{-2}$ & $1.58 \times 10^{-4}$ & $7.2 \times 10^{-3}$ \\
PANi-12b & $2.60 \times 10^{-3}$ & $5.81 \times 10^{-5}$ & $6.49 \times 10^{-5}$ \\
PANi-12a & $2.56 \times 10^{-3}$ & $1.55 \times 10^{-5}$ & $1.22 \times 10^{-5}$ \\
PANi-14 & $3.42 \times 10^{-3}$ & $1.25 \times 10^{-5}$ & $1.22 \times 10^{-5}$ \\
PANi-16 & $1.97 \times 10^{-4}$ & $6.32 \times 10^{-5}$ & $3.40 \times 10^{-4}$ \\
PANi-18 & $1.05 \times 10^{-3}$ & $4.12 \times 10^{-5}$ & $4.97 \times 10^{-4}$ \\
\hline
\end{tabular}

${ }^{\text {a }}$ Determined by four-probe technique.

chains or degree of $N$-alkylation increase. The MSAdoped series shows the best conductivity directly parallel to the highest doping level. The conductivities do not decrease when the side chains are long enough (18 for MSA, 12 for TSA and DBSA), and with DBSA, the conductivities start to even increase with the side chains of 16 and 18 carbons. These samples were the ones in which we found the highest degree of layering. It can be assumed that the formation of such ordering improves the extent of delocalization with a decrease in the ring-torsion.

It is well known that doping process generates defects (excitons, solitons, or (bi)polaronic sites) for the conjugated structure, which are essentially responsible for the electric conduction. The transport of electrons through three dimensions is expected to associated with interchain transfer and intrachain coherence length. ${ }^{25}$ The decrease in intrachain diffusion rate is reasonable for these $N$-alkylated polyanilines because of the defects caused by irregular deprotonation of amine sites during alkyl substitution. The optical spectra have reflected the twisting of the angle between phenyl ring and C$\mathrm{N}-\mathrm{C}$ plane due to increased steric repulsion from alkyl substituents. On the other hand, the electron-donating character of alkyl substituent on amine sites enhances the localization of charges along the polymeric chain. Additionally, the anisotropic orientation of conjugated chains can not only promote interchain diffusion of electrons but also optimize electron transportation within individual layer. It has been proved that the higher degree of order the conducting polymers have, the greater electrical conductivity they exhibit. ${ }^{4}$ Initial experimental evidence has already been obtained by Koide et al. ${ }^{26}$ The conductivity was shown to increase 10000 times as the liquid crystalline samples were annealed at nematic state. The liquid crystallinity was achieved by an attachment of mesogens covalently to a thiophene backbone. It is possible that the stabilization and somewhat improved conducting properties are assisted by the formation of the layered structures.

\section{CONCLUSION}

Through the use of three organic dopants with polyaniline derivatives, we have obtained understanding of ordering within the alkyl side chain substituted poly- 
anilines. The results from FT-IR and UV-Visible spectroscopies confirm the successful protonation of $N$ alkylated PANis by three dopants, MSA, TSA, and DBSA. The principle result is that the construction of layered structure is significantly affected by insertion of dopants into original layered neutral alkylated polyanilines. The cooperation of alkyl side chains and long alkyl tail of DBSA enables to develop well-defined layered structure. Interestingly DBSA seems to be able to stabilize doping at high extent due to long alkyl tail on the counterion. The interlayer distance $d$-spacing is confined by the length side chains or size of dopants without a decrease in the interdigitation. The alkyl side chain crystallization has been disrupted by the insertion of dopants, in particular with DBSA. The calculated domain size of layered phase $L$, representative of the perfection of the overall layer-ordered polymers is enlarged upon doping, including the impressive value up to $130 \AA$ because of the good cooperation of alkyl side chains and the alkyl tails of dopant counterions. The conductivity of the doped polymers decreases with the increasing length of side chains and degree of substitution but seems to actually increase under the conditions where layer formation has been achieved.

\section{REFERENCES}

1. J. W. Chevalier, J. Y. Bergeron, and L. H. Dao, Macromolecules, 25, 3325 (1992)

2. A. Watanabe, K. Mori, Y. Iwabuchi, Y. Iwasaki, Y. Nakamura, and O. Ito, Macromolecules, 22, 3521 (1989).

3. W. Y. Zheng, K. Levon, J. Laakso, and J. E. Österholm, Macromolecules, in press.

4. L. J. Buckley, D. K. Roylance, and G. E. J. Wnek, J. Polym. Sci., B, Polym. Phys., 25, 2179 (1987).
5. J. O. Nilsson and O. Inganäs, Synthetic Metals, 31, 359 (1989).

6. T. J. Prosa, M. J. Winokur, J. Mouton, P. Smith, and A. J. Heeger, Macromolecules, 25, 4364 (1992).

7. J. Moulton and P. Smith, Synth. Metals, 40, 13 (1991).

8. W. Wernet, M. Monkenbusch, and G. Wegner, Makromol. Chem., Rapid Commun., 5, 157 (1984).

9. Y. Cao, P. Smith, and A. J. Heeger, Synth. Metals, 48, 91 (1992).

10. K. Levon, G. Ho, W. Y. Zheng, J. Laakso, and J. Österholm, submitted to Polymer.

11. W. Y. Zheng, R. H. Wang, K. Levon, Z. Y. Rong, and T. Taka, submitted to Makromol. Chem.

12. G. Fredrickson, Macromolecules, 26, 2825 (1993).

13. Y. Cao, P. Smith, and A. J. Heeger, Synth. Metals, 55 -57, 3471 (1993).

14. Y. F. Nicolau and D. Djurado, Synth. Metals, 55-57, 394 (1993).

15. Z. H. Wang, A. Ray, A. G. MacDiarmid, and A. J. Epstein, Phys. Rev. B, 43, 4373 (1991).

16. M. J. Winokur, P. Wamsley, J. Moulton, P. Smith, and A. J. Heeger, Macromolecules, 24, 3812 (1991).

17. X. Q. Yang, J. Chen, P. D. Hale, T. Inagaki, T. A. Skotheim, D. A. Fischer, Y. Okamoto, L. Samuelsen, S. Tripathy, K. Hong, I. Watanabe, M. F. Rubner, and M. L. den Boer, Langmuir, 5 , 1288 (1989)

18. Y. Min, A. G. MacDiarmid, and A. Epstein, Polym. Prepr., Am. Chem. Soc., Piv. Polym. Chem., 231 (1994).

19. T. Kawai, M. Nakazono, and K. Yoshino, J. Mater. Chem., 2(9), 903 (1992).

20. J. P. Pouget, M. E. Józefowicz, A. J. Esptein, X. Tang, and A G. MacDiarmid, Macromolecules, 24, 779 (1991).

21. E. Stubb, E. Punkka, and J. Paloheimo, Mater. Sci. Eng., 10, 85 (1993).

22. S. K. Manohar, A. G. MacDiarmid, K. R. Cromack, J. M. Ginder, and A. J. Epstein, Synth. Metals, 29, E349 (1989).

23. X. Jing, J. Tang, Y. Wang, L. Lei, B. Wang, and F. Wang, Science in China, B, 33, 787 (1990).

24. J. R. Reynolds, A. D. Child, J. P. Ruiz, S. Y. Hong, and D. S. Marynick, Macromolecules, 26, 2095 (1993).

25. E. M. Genies, A. Boyle, M. Lapkowski, and C. Tsintavis, Synth. Metals, 36, 139 (1990).

26. N. Koide, Polym. Prepr., Jpn. (English Edition), 44, (1-5) E185 (1995). 